\title{
Application of Portable Near-Infrared Spectrometer for Analysis of Organic Carbon in Marine Sediments
}

\author{
Jing Yu' ${ }^{*}$, Jiangmei Mao',2, Tao Pan ${ }^{3}$, Pimao Chen1, Lijun Yao ${ }^{3 *}$ \\ ${ }^{1}$ South China Sea Fisheries Research Institute, Chinese Academy of Fishery Sciences/Key Laboratory of Fishery Ecology and \\ Environment, Guangdong Province/Scientific Observing and Experimental Station of South China Sea Fishery Resources and \\ Environment, Ministry of Agriculture/Key Laboratory of Marine Ranching Technology, CAFS, Guangzhou, China \\ ${ }^{2}$ Collage of Marine Science, Shanghai Ocean University, Shanghai, China \\ ${ }^{3}$ Department of Optoelectronic Engineering, Jinan University, Guangzhou, China \\ Email: *yujing@scsfri.ac.cn, *tylj@jnu.edu.cn
}

How to cite this paper: Yu, J., Mao, J.M. Pan, T., Chen, P.M. and Yao, L.J. (2017) Application of Portable Near-Infrared Spectrometer for Analysis of Organic Carbon in Marine Sediments. Journal of Geoscience and Environment Protection, 5, 108-114. https://doi.org/10.4236/gep.2017.55008

Received: April 20, 2017

Accepted: May 22, 2017

Published: May 25, 2017

Copyright (C) 2017 by authors and Scientific Research Publishing Inc. This work is licensed under the Creative Commons Attribution International License (CC BY 4.0).

http://creativecommons.org/licenses/by/4.0/

\section{Open Access}

\begin{abstract}
The performance of a portable near-infrared (NIR) spectrometer to determine organic carbon (OC) in marine sediments was evaluated. The NIR reflection spectra of 180 samples in the range 950 - $1650 \mathrm{~nm}$ were acquired using an ultra-compact spectrometer. NIR spectroscopy combined with the partial least squares (PLS) regression and Savitzky-Golay (SG) smoothing was successfully applied to rapid and reagent-free determination of OC. Using the PLS-SG model with $1^{\text {nd }}$ order derivative, $2^{\text {th }}$ polynomial and eleven smoothing points, the root-mean-square errors $\left(\mathrm{RMSEP}_{\mathrm{M}}\right)$ and correlation coefficients $\left(\mathrm{R}_{\mathrm{P}, \mathrm{M}}\right)$ of prediction for modeling were $0.073 \%$ and 0.894 , respectively, the root-meansquare errors $\left(\mathrm{RMSEP}_{\mathrm{V}}\right)$ and correlation coefficients $\left(\mathrm{R}_{\mathrm{P}, \mathrm{V}}\right)$ of prediction for validation were $0.075 \%$ and 0.883 , respectively. Results showed that the small portable NIR instrument achieved well prediction effect for the analysis of OC in marine sediments, which had advantages of rapid, easy to carry and operate suitable for large-scale applications to analyze marine sediments.
\end{abstract}

\section{Keywords}

Marine Sediments, Organic Carbon, Near-Infrared Spectroscopy, Portable Spectrometer

\section{Introduction}

Marine sediments preserve an abundance of climatic and environmental information for reconstructing oceanic history [1] [2]. Many of the traditional marine geologic and paleoceanographic techniques are usually slow and labor intensive, 
and performed in the laboratory, requiring the chemical reaction processes, not easy to operate.

Near-infrared (NIR) spectroscopy primarily reflects the absorption of overtones and the combination of the vibrations of $\mathrm{X}-\mathrm{H}$ functional groups (e.g., $\mathrm{C}-\mathrm{H}, \mathrm{O}-\mathrm{H}$, and $\mathrm{N}-\mathrm{H}$ ). Given the weak NIR absorption strength, most sample types can be directly measured without chemical reagents. In the geological samples, the NIR spectroscopy as a rapid and reagent-free measurement has been effectively utilized in soil analysis [3] [4] [5] [6] [7]. However, common to all these works is the fact that they employed benchtop spectrometers, and few studies have been reported on marine sediments, as far as we know. These benchtop spectrometers are bulky and expensive difficult to be large-scale application. With the advantages of rapid, easy to carry and operate, portable NIR instruments are very attractive for large-scale application, despite their reduced spectrophotometric characteristics.

Organic carbon (OC) is one of the major components in marine sediments and contains many hydrogen groups [8]. Several absorption bands of these groups exist in the NIR region. In this study, NIR spectroscopy of OC in marine sediments was directly measured by the small, dedicated and portable NIR spectrometer. Furthermore, NIR spectroscopy combined with the partial least squares (PLS) regression and Savitzky-Golay (SG) smoothing was applied to rapid and reagent-free determination of $\mathrm{OC}$ in marine sediments.

\section{Materials and Methods}

\subsection{Experimental Materials, Instruments, and Measurement Methods}

The marine sediment samples were collected from Daya Bay of China using a sediment corer and were cryopreservation at $-30^{\circ} \mathrm{C}$ for $5 \mathrm{~h}$, then $48 \mathrm{~h}$ for freeze drying in a lyophilizer, sieved through 100 mesh screen and stored in hermetic bag finally. Then, a total of 180 samples were obtained. Every sample was $50 \mathrm{~g}$. All samples were measured by the portable NIR spectrometer firstly, and then were determined through chemical method.

The OC values of these samples were measured with potassium dichromate redox titration [9], which is a capacity analysis method with potassium dichromate standard solution as a titrant. It requires adding potassium dichromate in the concentrated sulfuric acid medium and transforms OC in the sample to the $\mathrm{CO}_{2}$ by heating, where the remaining potassium dichromate was re-titrated using ferrous sulfate standard solution till to colour reaction. The quantification of the OC in the sample was indirectly measured by the consumption of potassium dichromate. Then the obtained values were provided as the reference values for spectroscopic analysis. The measured values ranged from 0.91 to 1.49 (\%) and the mean value and standard deviation were 1.09, 0.17 (\%), respectively, which were used as the reference values for the calibration modelling of NIR spectroscopic analysis.

A portable NIR spectrometer, the MicroNIR ${ }^{\mathrm{TM}}$ (JDSU Corporation, Milpitas, 
USA) Grating Spectrometer, which equips with a reflection accessory. This instrument, powered (5V) and controlled via USB port of a computer, originally employs two tungsten light bulbs as radiation source, a Linear Variable Filter (LVF) as dispersing element and an uncooled 128-element detector (InGaAs). An image of the MicroNIR spectrometer is provided in Figure 1. The scanning spectrum spanned $950 \mathrm{~nm}$ to $1650 \mathrm{~nm}$ with a range of $2 \mathrm{~nm}-4 \mathrm{~nm}$ wavelength gaps. Each sample was scanned ten times, and the mean value of the ten times was used for modeling. The spectra measured at $26^{\circ} \mathrm{C} \pm 1^{\circ} \mathrm{C}$ and $46 \pm 1$ (\%) RH.

\subsection{Calibration, Prediction and Validation Process}

A rigorous process of calibration, prediction and validation was performed to achieve parameter stability and objective evaluation for modeling.

In chronological order, the samples were divided into two groups. The first group (120 samples) was used for modeling, whereas the second group (60 samples) was used for validation. The second group excluded in modeling was used to validate and achieve an objective evaluation. The root-mean-square errors $\left(\mathrm{RMSEP}_{\mathrm{M}}\right)$ and correlation coefficients $\left(\mathrm{R}_{\mathrm{P}, \mathrm{M}}\right)$ of prediction for modeling were calculated. The calculation formulas are as follows:

$$
\begin{aligned}
& \operatorname{RMSEP}_{\mathrm{M}}=\sqrt{\frac{\sum_{i=1}^{n}\left(\tilde{C}_{i}-C_{i}\right)^{2}}{n}}, \\
& \mathrm{R}_{\mathrm{P}, \mathrm{M}}=\frac{\sum_{i=1}^{n}\left(C_{i}-C_{\mathrm{Ave}}\right)\left(\tilde{C}_{i}-\tilde{C}_{\mathrm{Ave}}\right)}{\sqrt{\sum_{i=1}^{n}\left(C_{i}-C_{\mathrm{Ave}}\right)^{2}\left(\tilde{C}_{i}-\tilde{C}_{\mathrm{Ave}}\right)^{2}}},
\end{aligned}
$$

where $n$ is the number of prediction samples; $C_{i}, \tilde{C}_{i}$ are the measured and predicted values for the $i^{\text {th }}$ prediction sample, respectively; and $C_{\text {Ave }}, \tilde{C}_{\text {Ave }}$ are the mean measured value and mean predicted value of all prediction samples, respectively. The model parameters were selected using the minimum $\operatorname{RMSEP}_{\mathrm{M}}$ to achieve good prediction accuracy. Finally, the selected model was validated

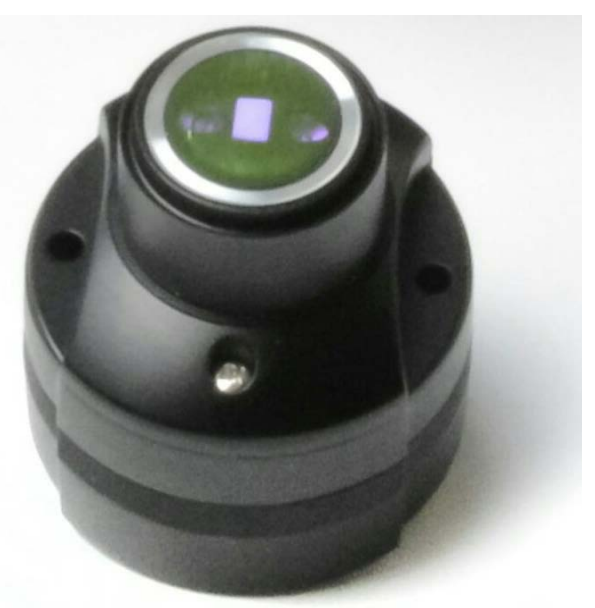

Figure 1. Map of the MicroNIR spectrometer. 
against the validation set (60 samples). The corresponding root-mean-square errors $\left(\mathrm{RMSEP}_{\mathrm{V}}\right)$ and correlation coefficients $\left(\mathrm{R}_{\mathrm{P}, \mathrm{V}}\right)$ of prediction for validation and were further calculated, respectively. The calculation formulas are as follows:

$$
\begin{aligned}
& \operatorname{RMSEP}_{\mathrm{V}}=\sqrt{\frac{\sum_{k=1}^{m_{\mathrm{V}}}\left(\tilde{C}_{\mathrm{V}, \mathrm{k}}-C_{\mathrm{V}, \mathrm{k}}\right)^{2}}{m_{\mathrm{V}}-1}}, \\
& \mathrm{R}_{\mathrm{P}, \mathrm{V}}=\frac{\sum_{k=1}^{m_{\mathrm{V}}}\left(C_{\mathrm{V}, \mathrm{k}}-C_{\mathrm{V}, \text { Ave }}\right)\left(\tilde{C}_{\mathrm{V}, \mathrm{k}}-\tilde{C}_{\mathrm{V}, \text { Ave }}\right)}{\sqrt{\sum_{k=1}^{m_{\mathrm{V}}}\left(C_{\mathrm{V}, \mathrm{k}}-C_{\mathrm{V}, \text { Ave }}\right)^{2} \sum_{k=1}^{m_{\mathrm{V}}}\left(\tilde{C}_{\mathrm{V}, \mathrm{k}}-\tilde{C}_{\mathrm{V}, \text { Ave }}\right)^{2}}},
\end{aligned}
$$

where $m_{\mathrm{V}}$ is the number of the validation samples; $C_{\mathrm{V}, \mathrm{k}}, \tilde{C}_{\mathrm{V}, \mathrm{k}}$ are the measured and predicted values of the $k^{\text {th }}$ validation sample; and $C_{\mathrm{V}, \text { Ave }}, \tilde{C}_{\mathrm{V}, \text { Ave }}$ are the mean measured value and mean predicted value of all validation samples.

\subsection{PLS Model with Savitzky-Golay Smoothing}

PLS method is widely used for spectroscopic analysis, and can screen spectroscopic data comprehensively and extract information variables [10] [11]. The number of PLS factors $(F)$ as an important parameter corresponds to the number of spectral integrated variables showing sample information. If the $F$ was set too small, the sample information in the spectra could not be fully used and the model accuracy would be decreased. If the $F$ was set too big, noises would be led into the model and the prediction ability would be decreased too. Therefore, it is very necessary to select reasonable $F$. In addition, the goal of spectral preprocessing is also to make full use of spectral information and to eliminate noise. Thus, combined with the spectral preprocessing, the optimization of the number of PLS factors will be more effective.

The NIR spectroscopy of marine sediment samples contains more physical and chemical noises. To overcome the noises, the measured spectra require pretreatment. SG smoothing is a useful spectrum pretreatment method with a wide range of applications and a variety of smoothing modes [12]. Based on the SG derivative spectra, the PLS model was established and has become an effective approach for improving analysis precision, as successfully used in many fields [5] [13] [14]. The parameters of SG smoothing include order of derivative (d), degree of polynomial $(p)$ and number of smoothing points ( $m$, odd). In the present study, the spectra of 180 marine sediment samples were pretreated with the SG smoothing mode $(d=1, p=2$ and $m=11)$. The computer platform was developed with MATLAB R2009b software.

\section{Results and Discussion}

The NIR spectra of the 180 marine sediments samples in the whole spectral collecting region (950 - $1650 \mathrm{~nm}$ ) are illustrated in Figure 2. As shown in Figure 2, the baseline drift of the NIR spectra is relatively large. 


\subsection{SG-PLS Models}

The SG derivative spectra of all marine sediment samples on the whole spectral collecting region are illustrated in Figure 3. As shown in Figure 3, shows that the baseline drift of the SG derivative spectra significantly improved. For the PLS model with the SG derivative spectra, the number of PLS factors $F, \operatorname{RMSEP}_{M}$ and $\mathrm{R}_{\mathrm{P}, \mathrm{M}}$ were $6,0.073 \%$ and 0.894 , respectively. The result indicated that the PLS model with SG smoothing achieved well prediction precision with the portable NIR spectrometer.

\subsection{Model Validation}

The validation samples excluded from the modeling process were used to validate the PLS model. The PLS regression coefficients were calculated using the spectra and measured values of all modeling samples depending on the corresponding parameters. The predicted OC values of the validation samples were then calculated using the obtained regression coefficients and the spectra of the validation samples.

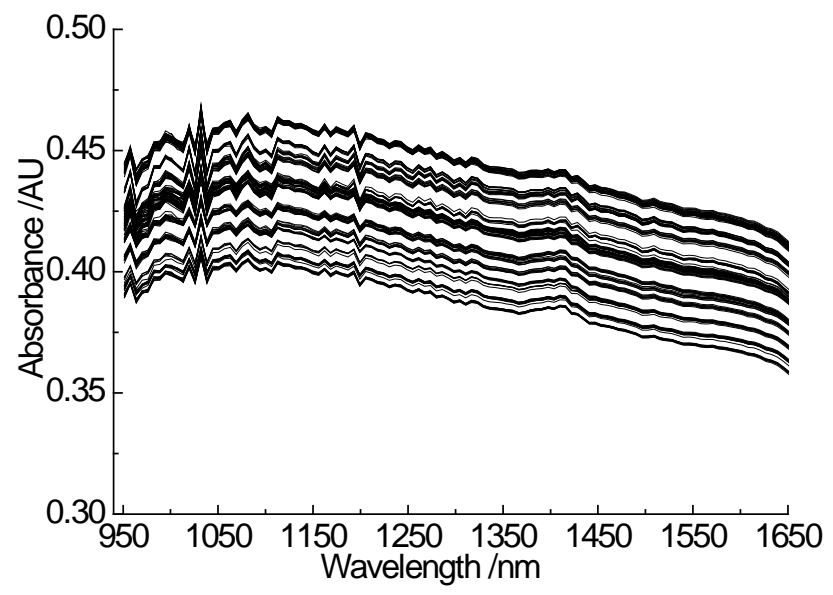

Figure 2. NIR spectra of the marine sediments samples (950 - 1650 $\mathrm{nm})$.

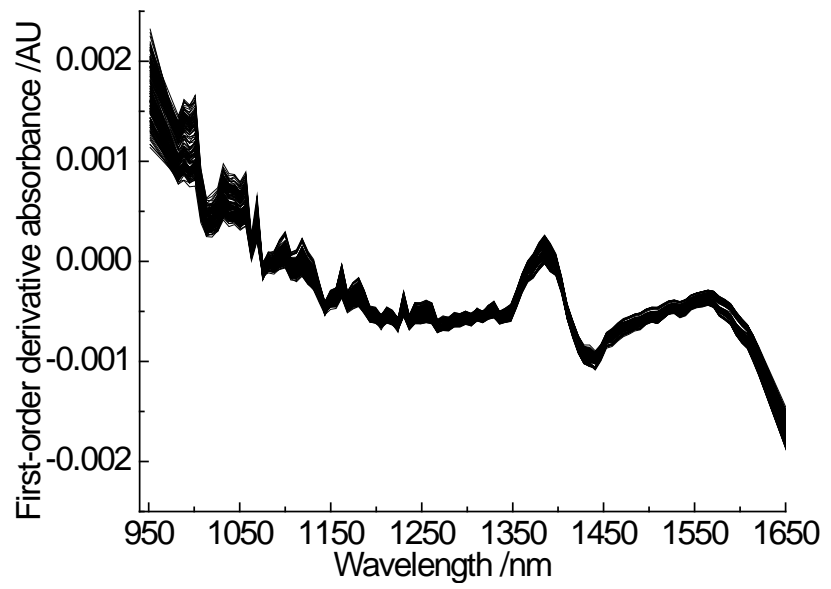

Figure 3. SG derivative spectra of the marine sediments samples with $1^{\text {nd }}$ order derivative, $2^{\text {th }}$ polynomial and eleven smoothing points. 


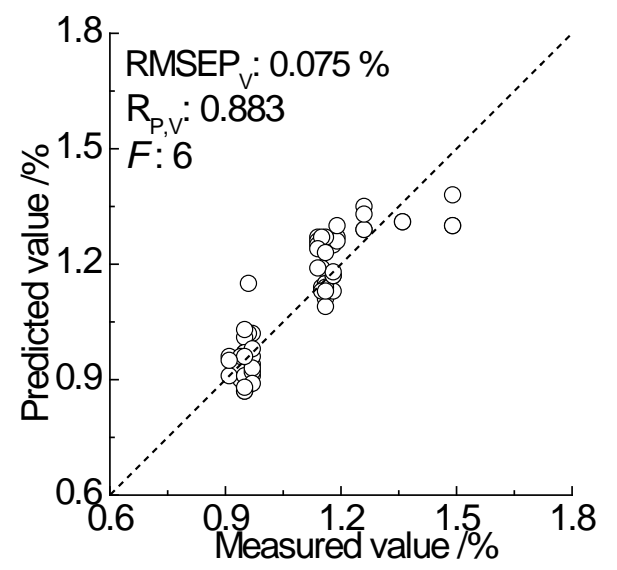

Figure 4. Relationship between the predicted and measured OC values of the validation samples.

The relationship between the predicted and measured OC values of the validation samples are shown in Figure 4. The RMSEP ${ }_{V}$ and $R_{P, V}$ were $0.075(\%)$ and 0.883 , respectively, which achieved well validation effect.

\section{Conclusion}

In this paper, the marine sediments samples were detected by the small portable NIR instrument and the NIR spectroscopy combined with PLS method and SG smoothing was successfully applied to reagent-free and rapid determination of OC in marine sediments and achieved well validation effect. Results showed that the small portable NIR instrument achieved well prediction effect for the analysis of OC in marine sediments, which had advantages of rapid, easy to carry and operate suitable for large-scale applications to analyze marine sediments.

\section{Acknowledgements}

This work was supported by National Key Technology Support Program (No. 2012BAD18B01, 2012BAD18B02), the Science and Technology Project of Shenzhen of China (No.JSGG20141015154342147, JCYJ20160331141759795), and the National Special Fund for Agroscientific Research in the Public Interest of China (No. 201003068).

\section{References}

[1] Chang, C.W., You, C.F., Huang, C.Y. and Lee, T.Q. (2005) Rapid Determination of Chemical and Physical Properties in Marine Sediments Using a Near-Infrared Reflectance Spectroscopic Technique. Applied Geochemistry, 20, 1637-1647. https://doi.org/10.1016/j.apgeochem.2005.04.011

[2] Gu, Y.G., Lin, Q., Wang, Z.H., Ma, S.W., Lu, T.T., Ke, C.L. and Du, F.Y. (2013) Spatial Distribution and Potential Ecological Risk Assessment of Heavy Metals in Zhelin Bay Sediments and Its Vicinity, China. South China Fisheries Science, 9, 32-37.

[3] Cozzolino, D. and Moron, A. (2006) Potential of Near-Infrared Reflectance Spectroscopy and Chemometrics to Predict Soil Organic Carbon Fractions. Soil Tillage Research, 85, 78-85. https://doi.org/10.1016/j.still.2004.12.006 
[4] Rossel, R.A.V., Walvoort, D.J.J., McBratney, A.B., Janik, L.J. and Skjemstad, J.O. (2006) Visible, Near Infrared, Mid Infrared or Combined Diffuse Reflectance Spectroscopy for Simultaneous Assessment of Various Soil Properties. Geoderma, 131, 59-75. https://doi.org/10.1016/j.geoderma.2005.03.007

[5] Chen, H.Z., Pan, T., Chen, J.M. and Lu, Q.P. (2011) Waveband Selection for Nir Spectroscopy Analysis of Soil Organic Matter Based on Sg Smoothing and MWPLS Methods. Chemometrics Intelligent Laboratory Systems, 107, 139-146. https://doi.org/10.1016/j.chemolab.2011.02.008

[6] Pan, T., Li, M.M. and Chen, J.M. (2014) Selection Method of Quasi-Continuous Wavelength Combination with Applications to the Near-Infrared Spectroscopic Analysis of Soil Organic Matter. Applied. Spectroscopy, 68, 263-271. https://doi.org/10.1366/13-07088

[7] Pan, T., Han, Y., Chen, J.M., Yao, L.J. and Xie, J. (2016) Optimal Partner WaveLength Combination Method with Application to Near-Infrared Spectroscopic Analysis. Chemometrics Intelligent Laboratory Systems, 156, 217-223. https://doi.org/10.1016/j.chemolab.2016.05.022

[8] Luo, Z.L., Zhu, C.B., Guo, Y.J., Su, L., Li, J.W. and Ou, Y.J. (2014) Distribution Characteristics of $\mathrm{C}$ and $\mathrm{P}$ in Liusha Bay Surface Sediment and Their Pollution Assessment. South China Fisheries Science, 10, 1-8.

[9] National Standards of the People's Republic of China (1998) The Specification for Marine Monitoring Part 5: Sediment Analysis.

[10] Burns, D.A. and Ciurczak, E.W. (2001) Handbook of Near-Infrared Analysis. 2nd Edition, Marcel Dekker Incorporation, New York.

[11] Lu, W.Z. (2007) Modern Near-Infrared Spectroscopy Analytical Technology. 2nd Edition, China Petrochemical Press, Beijing.

[12] Savitzky, A. and Golay, M.J.E. (1964) Smoothing and Differentiation of Data by Simplified Least Squares Procedures. Analytical Chemistry, 36, 1627-1637. https://doi.org/10.1021/ac60214a047

[13] Guo, H.S., Chen, J.M., Pan, T., Wang, J.H. and Cao, G. (2014) Vis-NIR Wavelength Selection for Non-Destructive Discriminant Analysis of Breed Screening of Transgenic Sugarcane. Analytical Methods, 6, 8810-8816. https://doi.org/10.1039/C4AY01833H

[14] Liu, Z.Y., Liu, B., Pan, T. and Yang, J.D. (2013) Determination of Amino Acid Nitrogen in Tuber Mustard Using Near-Infrared Spectroscopy with Waveband Selection Stability. Spectrochimica Acta Part A: Molecular and Biomolecular Spectroscopy, 102, 269-274. https://doi.org/10.1016/j.saa.2012.10.006 
Submit or recommend next manuscript to SCIRP and we will provide best service for you:

Accepting pre-submission inquiries through Email, Facebook, LinkedIn, Twitter, etc. A wide selection of journals (inclusive of 9 subjects, more than 200 journals)

Providing 24-hour high-quality service

User-friendly online submission system

Fair and swift peer-review system

Efficient typesetting and proofreading procedure

Display of the result of downloads and visits, as well as the number of cited articles Maximum dissemination of your research work

Submit your manuscript at: http://papersubmission.scirp.org/

Or contact gep@scirp.org 